\title{
Influence of Permeability Behavior on the Seepage Field and Stability of Reservoir Landslide
}

\author{
Chongyang Qiu ${ }^{1, a^{*}}$, Fei Sun ${ }^{1, b}$, Aiming Liao ${ }^{1, c}$ and Quansheng Deng ${ }^{1, d}$ \\ ${ }^{1}$ Hubei yichang Three Gorges Universityr, in China

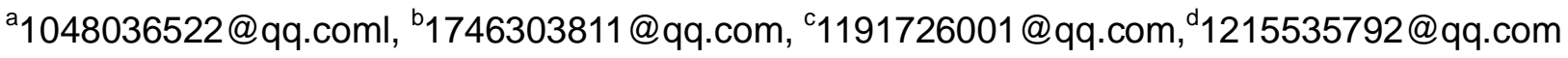

\begin{abstract}
Keywords: landslide; permeability coefficient; seepage field; stability coefficient
Abstract. The changes of underwater level have great impacts on the seepage field and the stability of reservoir landslide. The permeability coefficient is the principal factor that impacts on the changes of underwater. Based on the landslide in Three Gorges Reservoir area, the computing model was established to analyze the change law of seepage field and stability with the change of permeability coefficient. The results show that when the permeability coefficient is relatively large, with gradual increase of the degree of submersed slip mass, its stability decrease gently and then sharply. When the permeability coefficient is moderate, its stability keep constant and then decrease slightly. When the permeability coefficient is small, its stability increase dramatically and then gently.
\end{abstract}

\section{Introduction}

Reservoir landslide is one of the major geological disasters. The rise and fall of reservoir water levels change the seepage field of landslide which is closely relate to the permeability coefficient of landslide, and affects its stability. There are many geological disasters in the Three Gorges Reservoir where the reservoir water levels periodically fluctuate between $145 \mathrm{~m}$ and $175 \mathrm{~m}$. The fall of water level fluctuation is $30 \mathrm{~m}$. The studying different permeability and discussing the change properties of seepage field and stability of reservoir landslide have significant to it's prevention and treatment.

\section{The influence of permeability coefficient on the seepage field of landslide}

Seepage computing model

Based on a landslide in Three Gorges Reservoir area, the landslide is ancient rock landslide consisted of layered quartz sandstone and silt block crack rock in Jurassic system under the xiangxi formation. The landslide bed consists of two rocks strata. Bedding consists of medium bed carbonaceous siltstone at the bottom of xiangxi formation and cutting bed is made up of shape quartz sandstone in the middle of xiangxi formation. In the seepage computing model ,the water level fluctuation on the underground water is mainly considered. The model is established, shown in Fig.1, which divided into 13412 elements and 13233 joints.

Seepage computing scheme

Considering the normal reservoir storage is $175 \mathrm{~m}$ in Three Gorges, the water level is periodically fluctuate from $145 \mathrm{~m}$ to $175 \mathrm{~m}$. The velocity of water level is $1.0 \mathrm{~m} / \mathrm{d}$ and continuously run for thirty days until the end of December, the reservoir level rises to $175 \mathrm{~m}$, then the reservoir level maintain for ninety days, then drops to $145 \mathrm{~m}$. The relations between water level elevation $(\mathrm{H}(\mathrm{t}))$ and time $(\mathrm{t})$ is founded as follow, shown in Fig. 2. 


$$
H(t)=\left\{\begin{array}{l}
145+t, \quad t \in(0,30 d) \\
175, \quad t \in(30,90 d)
\end{array}\right.
$$

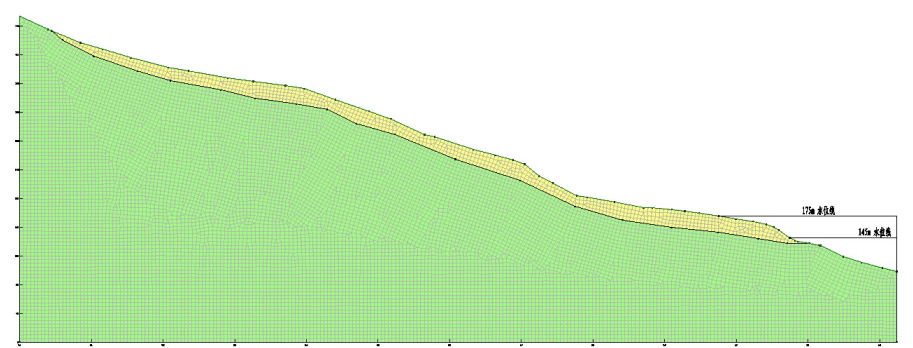

Fig. 1 The finite element grid of seepage computation

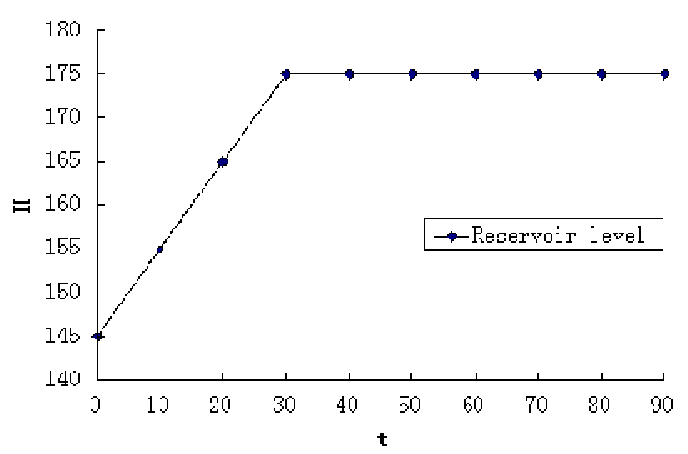

Fig.2 The curves of reservoir water level

Seepage computing parameters

In order to study the saturation line of underground water under different permeability behavior, according to the rank criterion of rock and soil permeability in the code for engineering geological investigation, choosing six order of magnitudes from four permeability ranks to compute the seepage of slip mass under the condition of reservoir water fluctuation, shown in table 1.

Table 1 The classification of landslide permeability

\begin{tabular}{|c|c|c|}
\hline Calculation type & Permeability coefficient $\mathrm{k}(\mathrm{m} / \mathrm{s})$ & Permeability rank \\
\hline 1 & $1.0 \times 10^{-2}$ & strong water permeability \\
\hline 2 & $1.0 \times 10^{-3}$ & strong water permeability \\
\hline 3 & $1.0 \times 10^{-4}$ & strong water permeability \\
\hline 4 & $1.0 \times 10^{-5}$ & medium water permeability \\
\hline 5 & $1.0 \times 10^{-6}$ & medium water permeability \\
\hline 6 & $1.0 \times 10^{-7}$ & weak water permeability \\
\hline
\end{tabular}

The analysis of seepage computing

In the rising stage of reservoir water level, with different permeability coefficient $\left(\mathrm{k}=1.0 \times 10-3 \mathrm{~m} / \mathrm{s}-1.0 \times 10^{-8} \mathrm{~m} / \mathrm{s}\right)$, during 90 days the distribution laws of underground water saturation line in the landslide was plotted.(Fig. 3-7)

(1) The permeability behavior of landslide is the principal factor that affects the seepage distribution. The shape character of seepage field depends on permeability behavior of rock and earth mass, when the reservoir level rise to a certain elevation and the permeability coefficient is larger, the smaller the angle between relative saturation line and horizontal negative direction(Fig.3), vice versa.

(2) The relation between the saturation line and reservoir water. When the permeability coefficient is lager $\left.>1.0 \times 10^{-4} \mathrm{~m} / \mathrm{s}\right)$, the change of saturation line keep pace with the change of reservoir water level. When the permeability coefficient is relatively small $\left(<1.0 \times 10^{-4} \mathrm{~m} / \mathrm{s}\right)$, the change of saturation line lag behind the change of reservoir water level, and the reservoir water tend to 'backward' the slope. When the reservoir water reach the highest point, with the increase of time, the effect of 'backward' gradually die away until disappear. 


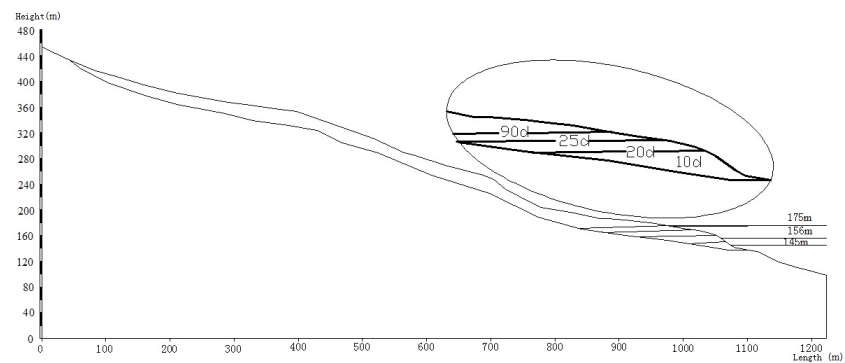

Fig. $3 \mathrm{k}=1.0 \times 10^{-3} \mathrm{~m} / \mathrm{s}(0-90 \mathrm{~d})$ Landslide groundwater seepage line

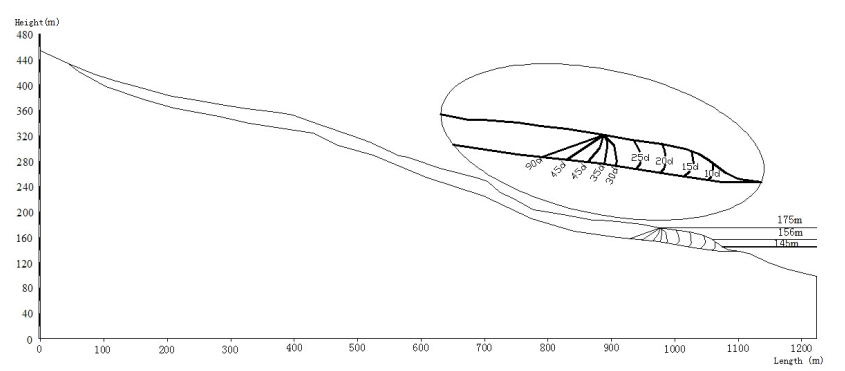

Fig.5 k=1.0 $\times 10^{-5} \mathrm{~m} / \mathrm{s}(0-90 \mathrm{~d})$ Landslide groundwater seepage line

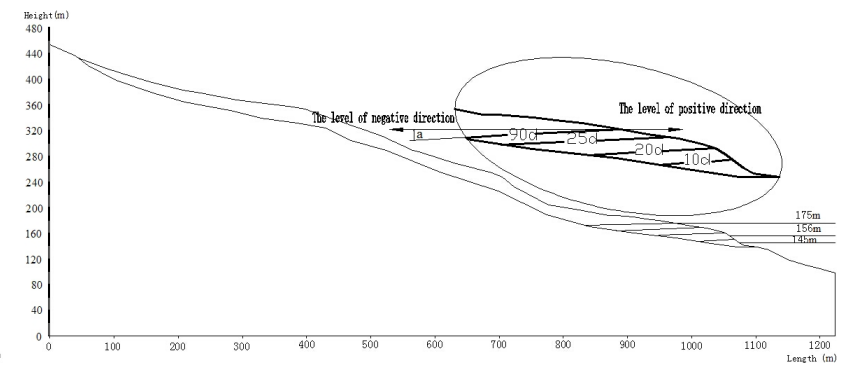

Fig. $4 \quad \mathrm{k}=1.0 \times 10^{-4} \mathrm{~m} / \mathrm{s}(0-90 \mathrm{~d})$ Landslide groundwater seepage line

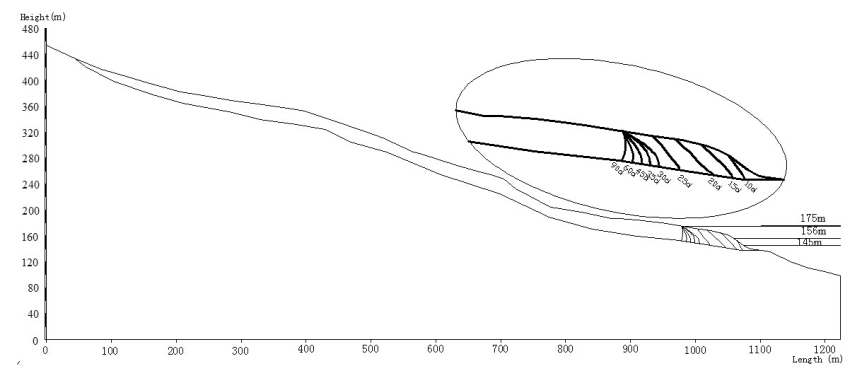

Fig6 k=1.0 $\times 10^{-6} \mathrm{~m} / \mathrm{s}$ (0-90d) Landslide groundwater seepage line

\section{Effect of wade degree on the stability of the landslide}

The wading degree of landslide is also a important factor which influence the stability of landslide, and define wading degree parameters for $\mathrm{s}$ 。

$$
s=\frac{\Delta h}{\Delta H}
$$

In the formula(2), $\Delta \mathrm{h}$ — the reservoir slope waterhead of landslide surface,the unit is $\mathrm{m}$. $\Delta \mathrm{H} —$ the height of the landslide, that is elevation difference of leading edge and trailing edge, the unit is $\mathrm{m}$ 。

The wading degree parameter range is 0.034 to 0.135 , seven kinds of working condition of wading $(0.034,0.04,0.06,0.08,0.10,0.12,0.135)$ are designed, taking the permeability coefficient of landslide $\mathrm{k}$ for $1.0 \times 10^{-3} \mathrm{~m} / \mathrm{s}, 1.0 \times 10^{-5} \mathrm{~m} / \mathrm{s}$ and $1.0 \times 10^{-7} \mathrm{~m} / \mathrm{s}$, analyzing the relationship between the degree of wading and landslide stability. The results are shown in Fig.8-10.
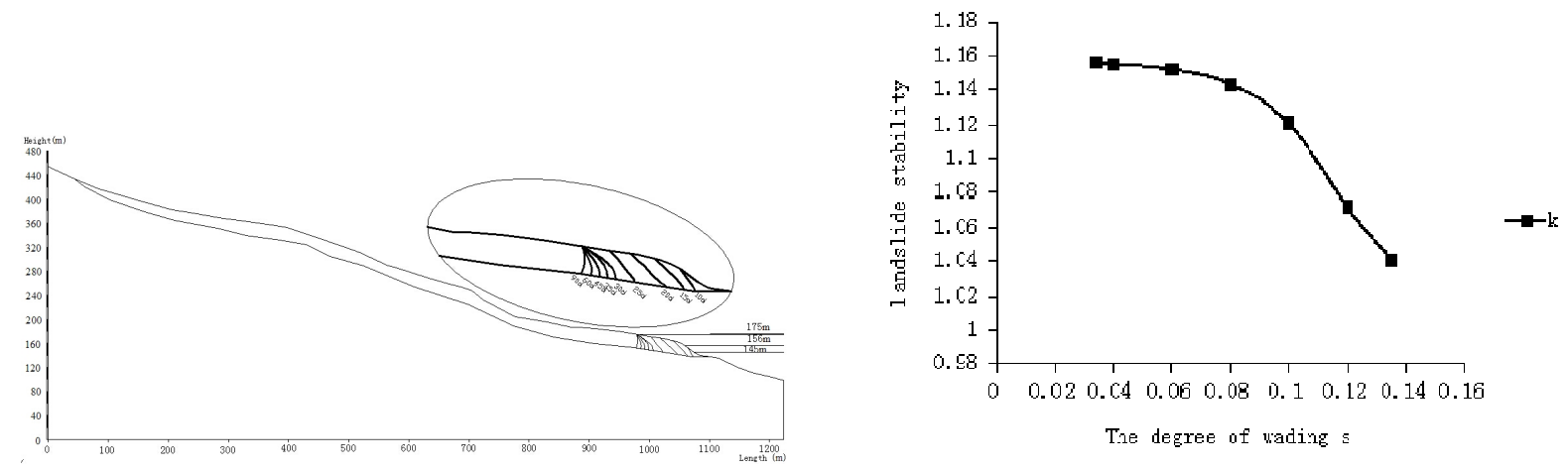

Fig. 7 k=1.0 $\times 10^{-7} \mathrm{~m} / \mathrm{s}$ （0-90d） Landslide Fig.8 Floating decrease heavy landslide stability groundwater seepage line degree of wading $\left(\mathrm{k}=1.0 \times 10^{-3} \mathrm{~m} / \mathrm{s}\right)$ 


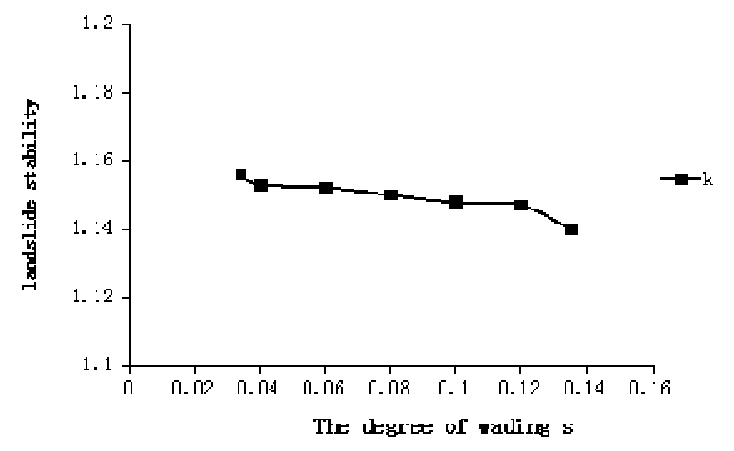

Fig.9 Mixed landslide stability with different stability degree of wading $\left(\mathrm{k}=1.0 \times 10^{-5} \mathrm{~m} / \mathrm{s}\right)$

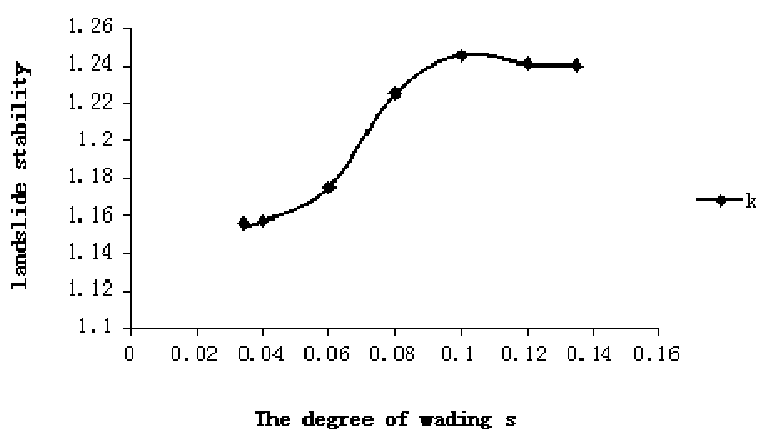

Fig.10 Dynamic water pressure type landslide with different degree of wading $\left(\mathrm{k}=1.0 \times 10^{-7} \mathrm{~m} / \mathrm{s}\right)$

Fig.8 shows that when landslide permeability coefficient is larger, with the wading degree increasing gradually, the stability of landslide reduces slowly at first, and then has a rapid trend to decrease. This is because of the reservoir water has a process of infiltration to the landslide, underground water seepage line is low changing at first, the floating effect scope of sliding resistance segment is small, the landslide stability decreases slowly, and when underground water seepage line has reached a certain height, the floating effect scope of sliding resistance segment is large, the landslide stability decreased rapidly.

Fig.9 shows that when the permeability coefficient is moderate, with gradual increase of the degree of submersed slip mass, the landslide stability keep constant and then decrease slightly. With the degree of wading gradual increasing, reservoir water has a deep supply to the underground water in the landslide, but the rise of underground water in the landslide slightly lags behind the reservoir water level rising, below the reservoir water line(vertical direction) landslide exists floating force effect which is produced by the hydrostatic pressure, so the landslide above water seepage line (vertical direction) combined with the floating force effect by the hydrostatic pressure and the seepage force effect by the dynamic water pressure, with infiltration deepen, floating force gradually increased and stability decreased gradually.

Fig. 10 shows that when the permeability coefficient is small, landslide stability with the wading degree increases gradually speeding up at first and then trend to increase slowly. This is because of the initial stage of reservoir water can only carry on the shallow underground water in the landslide supplies, failed to timely replenishment of deep water, so as to make between reservoir water and the underground water seepage line in the landslide body form pressure difference, and forming seepage pointing to the body, the reservoir water has a counter pressure effect which is favorable to the landslide stability.

\section{Conclusion}

(1)The permeability coefficient has a great influence on the position and the change of the underground water level.

(2)when the permeability coefficient is relatively large. with gradual increasing of the degree of submersed slip mass, the landslide stability decrease gently and then sharply.

(3) When the permeability coefficient is moderate, with gradual increase of the degree of submersed slip mass, the landslide stability keep constant and then decrease slightly.

(3) When the permeability coefficient is small, with gradual increase of the degree of submersed slip mass, the landslide stability increase dramatically and then gently. 


\section{Acknowledgements}

This study was supported by the scientific research project of Hubei Province(2012FFA040).

\section{Reference}

[1]Guangrun Liu,Echuan Yan:Resources Environment and Engineering,2005,03:147-149.(in chinese)

[2]Shirong Xiao,Zhiyu Hu,Shusheng Lu,Chengtao Ming:Journal of Yangtze River Scientific Research Institute,2013,11:39-44.(in chinese)

[3]Taoyi Luo.The research of Landslide Deformation Mechanism Induced by reservoir Water Changes and Criterion of Early Warning[D].Chengdu Univerisity of Technology ,2014.(in chinese)

[4]Ran Hu,Yifeng Chen, Chuangbing Zhou:Chinese Journal of Rock Mechanics and Engineering,2013,06:1279-1287.(in chinese)

[5]Caihua Liu,Congxin Chen,Xiating Feng:Rock and Soil Mechanics,2005,05:769-773.(in chinese)

[6]Xinhua xue,Xingguo Yang,Xin Chen.Application of a support vector machine for prediction of slope stability[J]. Science China(Technological Sciences),2014,12:2379-2386.

[7]Tao zheng, Yudeng Zhang,Xinsheng Mao:Journal of Water Resources and Architectural Engineering,2008,01:6-8+33.(in chinese)

[8]Shangjun Cai.Influence of Rising and falling of a River Water Level on Groundwater Flow in a Lanslide and Stability of a Lanslide[D].Chengdu Univerisity of Technology,2013.(in chinese)

[9]Dapeng Zhu. Revival Mechanism and Deformation Prediction of Typical Accumulative Landslide in the Three Gorges Reservoir[D].China University of Geosciences,2010.(in chinese) [10]Daipeng Zhao,Shimei Wang,Yunzhi Tan,Qinghua Zhan:Rock and Soil Mechanics,2013,04: 1017-1024.(in chinese) 